\title{
Down-regulation of fractalkine inhibits the in vitro and in vivo angiogenesis of the hepatocellular carcinoma HepG2 cells
}

\author{
FENG LI, ZUOREN WANG, YONGCUN LIU and JUNHUI LI \\ Department of Hepatobiliary Surgery, the First Affiliated Hospital of Medical College, \\ Xi'an Jiao Tong University, 277 West Yanta Road, Xi'an 710061, Shaanxi Province, P.R. China
}

Received January 12, 2010; Accepted March 18, 2010

DOI: 10.3892/or_00000906

\begin{abstract}
There is increasing evidence that hepatocellular carcinoma (HCC) is inherently associated with the inflammatory process and the up-regulation of cytokines. Our study aimed at elucidating the role of the cytokine fractalkine in the process of HCC carcinogenesis. Expression of fractalkine in hepatocellular carcinoma cell line HepG2 was knocked-down by RNAi. Conditioned media (CMs) from HepG2 was used in angiogenesis assays both in vitro and in vivo. Compared with CMs from mock transfection and negative shRNA treated HepG2, CMs from fractalkine shRNA treated HepG2 highly suppressed the migration, proliferation, and differentiation of human umbilical vein endothelial cells. The results suggest that fractalkine may play a key role in the mechanism of angiogenesis and carcinogenesis of HCC.
\end{abstract}

\section{Introduction}

Hepatocellular carcinoma (HCC) is the fifth most common malignancy in the world, leading to an estimated one million deaths annually (1). Although several treatment options are available, including surgical resection and transcatheter arterial embolization, the prognosis for HCC patients remains poor (2). Liver cancers are highly vascularized, and experimental and clinical data indicate that the progression of cancer is associated with increased angiogenesis, and that an increase in microvascular density is associated with a poor progression (3). This suggests that inhibition of angiogenesis may be an effective treatment for HCC.

Angiogenesis, the formation of new blood vessels, is essential for the survival of tumors. The first step in angiogenesis may be the secretion of angiogenic factors, which are produced by the cancer cells themselves $(4,5)$, and by infiltrating inflammatory cells (6). These factors promote the formation of new vessels, which can sprout from pre-existing

Correspondence to: Dr Zuoren Wang, Department of Hepatobiliary Surgery, the First Affiliated Hospital of Medical College, Xi'an Jiao Tong University, 277 West Yanta Road, Xi'an 710061, Shaanxi Province, P.R. China

E-mail: wangzuor@yahoo.com.cn

Key words: fractalkine, angiogenesis, hepatocellular carcinoma, RNAi vessels, or can develop from circulating endothelial cells derived from blood vessel walls. The angiogenic process is controlled by a wide variety of positive and negative regulators, which include growth factors, cytokines, adhesion molecules, and chemokines.

Chemokines are a large family of chemoattractant cytokines which are initially recognized for their ability to regulate leukocyte trafficking and homing $(7,8)$. They are classified into four groups: CXC, CC, C and CX3C, based on the number and location of the cysteine residues at the $\mathrm{N}$ terminus of the molecule $(7,8)$. Recent studies have shown that this family participates in the development of cancers (9). Fractalkine is the only CX3C chemokine. It contains three amino acids between the first two cysteine residues and exists as two functional forms: soluble and membrane-bound $(10,11)$. The membrane-anchored form of fractalkine consists of a chemokine head tethered to the cell surface by a mucin-like stalk, followed by a single trans-membranespanning domain. Fractalkine is expressed on endothelial cells, dendritic cells, and intestinal epithelial cells (12-14). CX3CR1, the unique receptor of fractalkine, belongs to a family of specific $\mathrm{G}$ protein-coupled seven-transmem-brane domain receptors, and is expressed on $\mathrm{T}$ cells, natural killer cells, monocytes, and microvascular endothelial cells (15). Many studies have suggested that chemokines and their receptors are involved in the recruitment of leukocytes and in mediating the antitumor immune responses. An increasing number of studies has also shown that the interactions between chemokines and their receptors play an important role in regulating tumor pathogenesis and progression.

Fractalkine functions as an adhesion molecule, allowing communication between tumor cells and stromal cells. Accumulating evidence suggests that fractalkine is involved in the pathogenesis of various types of cancers, particularly liver cancer. However, the precise role of fractalkine in hepatocellular carcinoma is unclear. It has been suggested that fractalkine participates in chronic inflammation and rheumatoid arthritis, by stimulating angiogenesis, via the activation of endothelial cells (16). Fractalkine in synovial fluid from patients with rheumatoid arthritis has been found to promote angiogenic activity in vitro. In addition, patients with proliferative diabetic retinopathy have been found to have elevated levels of fractalkine in the vitreous humor (17). Fractalkine can also induce the migration of endothelial cells and the formation of endothelial cell capillary tubes on a synthetic matrix. One study has shown that fractalkine induced more 
Table I. Three pairs of two single-stranded DNA oligonucleotides.

\begin{tabular}{lll}
\hline Target site & \multicolumn{1}{c}{ Top strand } & \multicolumn{1}{c}{ Bottom strand } \\
\hline ShRNA-78 & CACCACGGTGTGACGAAATGCAATCAAG & GATCCAAAAAAACGGTGTGACGAAATGC \\
& AGTTGCATTTCGTCACACCGTTTTTTG & AACTCTTGATTGCATTTCGTCACACCGT \\
ShRNA-1153 & CACCGAGCTGTGGTAGTAATTCATCAAG & GATCCAAAAAAGAGCTGTGGTAGTAATT \\
& AGTGAATTACTACCACAGCTCTTTTTG & CACTCTTGATGAATTACTACCACAGCTC \\
ShRNA-3154 & CACCGGATGATCTAGGAAGACTTTCAAG & GATCCAAAAAAGGATGATCTAGGAAGAC \\
& AGAAGTCTTCCTAGATCATCCTTTTTG & TTCTCTTGAAAGTCTTCCTAGATCATCC \\
\hline
\end{tabular}

blood vessel growth than controls in the rabbit corneal pocket neovascularization assay. We hypothesize that up-regulation of fractalkine promotes angiogenesis in liver cancer through the binding of its receptor CX3CR1. In this study we used RNAi to knock down the expression of fractalkine in the hepatocellular carcinoma cell line hepG2, in order to study its function.

\section{Materials and methods}

Cells and reagents. The hepatocellular carcinoma cell lines HepG2, Hep3B and the endothelial cell line HUVEC were purchased from the American Type Culture Collection (Washington DC, USA). The cell lines SMMC7721, MHCC97H and the normal liver cell line L02 were purchased from the Chinese Academy of Sciences (Shanghai, China). They were stored in the Department of Hepatobiliary Surgery, at the First Affiliated Hospital of Xi'an Jiao Tong University. Cells were cultured in DMEM medium (Hyclone) supplemented with 10\% FBS (Hyclone), penicillin $(100 \mathrm{U} / \mathrm{ml})$ and streptomycin $(0.1 \mathrm{mg} / \mathrm{ml})$ at $37^{\circ} \mathrm{C}$ in a humidified atmosphere of $95 \% \mathrm{O}_{2}$ and $5 \% \mathrm{CO}_{2}$. G418 was purchased from Invitrogen (Invitrogen, CA). A human fractalkine quantitative ELISA kit from B\&C Science (Shanghai, China) was used to detect soluble fractalkine following the manufacturer's instruction.

Fractalkine shRNA expression vector construct. The human fractalkine gene sequence (Genbank accession NM_002996) was analyzed for a potential shRNA target, using the webbased shRNA target finder and design tools provided by Invitrogen according to the manufacturer's instruction. Three shRNA insert sequences were synthesized (Table I). As a negative control, a vector was also designed, with the inserted sequence 5'-caccGTTCTCCGAACGTGTCACGT caagagattACGTGACACGTTCGGAGAAttttttg-3', which does not target any region in the human genome. After annealing of the sense and antisense oligonucleotides and digestion, the fragments were inserted into the shRNA expression vector pGPU6/GFP/Neo (Genepharma Corp. Shanghai, China) and positive clones were confirmed by plasmid sequencing.

Transfection of fractalkine shRNA expression vector. Cells were plated in 24 -well plates $\left(3 \times 10^{4}\right.$ cells per well) one day before transfections, which were carried out using lipofectamine 2000 (Invitrogen, CA) according to the manufacturer's protocol. After $24 \mathrm{~h}$, cultures were diluted at 1:10 and regenerated. Selection of adherent cells was performed by the addition of G418. A limited dilution was used in 96-well plates for repeated colony selections. After 14 days, $\mathrm{G} 418(200 \mu \mathrm{l} / \mathrm{ml})$ was added.

Reverse transcriptase polymerase chain reaction. Total RNA was isolated using TRIzol reagent according to the manufacturer's instructions (Invitrogen, CA) and converted to cDNA by incubation with AMV reverse transcriptase. The polymerase chain reaction was performed using specific primer pairs for fractalkine (5'-GCCACCTTCTGCCATC TGACTG-3' and 5'-TCCTTGACCCATTGCTCCTTCG-3'). The housekeeping gene $B$-actin (5'-ATCGTGCGTGACATT AAGGAGAAG-3' and 5'-AGGAAGGAAGGCTGGAAGA GTG-3') was used as an internal control.

Western blot analysis. Cells were lysed in ice-cold RIPA buffer with protease inhibitors. Whole cell lysates were obtained by subsequent centrifugation at $12000 \mathrm{rmp}$ for $15 \mathrm{~min}$ at $4{ }^{\circ} \mathrm{C}$. Protein concentrations were determined by the Bradford assay (Bio-Rad, CA), using the bovine serum albumin as the standard. Cell extracts were electrophoretically resolved on a denaturing SDS polyacrylamide gel and transferred to a nitrocellulose membrane. The membranes were initially blocked with $5 \%$ non-fat dry milk in Trisbuffered saline (TBS) for $2 \mathrm{~h}$ and then probed with primary antibodies against the specific protein and $B$-actin (internal control). After incubation with the primary antibodies at $4^{\circ} \mathrm{C}$ over-night, the membrane were hybridized with the secondary goat anti-rabbit antibody (1:2000) or goat antimouse anti-body (1:2000) (Santa Cruz, CA) for $2 \mathrm{~h}$ at room temperature. The immunopositive bands were examined by an enhanced chemiluminescence detection system (Amersham Bioscience, NJ). All analyses were performed in duplicate. The anti-bodies used were fractalkine (1:1000) (Ebioscience, CA) and $\beta$-actin (1:1000) (Santa Cruz).

MTT assay. The methylthiazolyl tetrazolium (MTT) test was used to evaluate the effect of various conditioned media on endothelial cell proliferation. HUVEC was cultured in standard medium, trypsinized, and seeded at $1 \times 10^{3}$ cells/well in triplicate on 96-well plates and incubated at $37^{\circ} \mathrm{C}$ for $24 \mathrm{~h}$, to allow cells to attach. The media were then removed and replaced with $200 \mu 1$ conditioned media: i) mock trans- 
fection conditioned medium; ii) negative shRNA transfection conditioned medium; iii) fractalkine shRNA transfection conditioned medium. After $24 \mathrm{~h}, 20 \mu \mathrm{l}$ MTT was added to each well and incubation was allowed to continue for another $4 \mathrm{~h}$. Finally, $100 \mu \mathrm{l}$ DMSO was added to each well. The absorption value was measured (18).

Migration assay. The effect of conditioned media on endothelial cell migration was measured using the BD BioCoat Matrigel invasion chamber and following the manufacturer's instructions. Briefly, confluent monolayers of HUVEC were cultured with media containing no growth factor for $12 \mathrm{~h}$ before harvesting. Harvested cells were suspended in serumfree DMEM and the HUVEC suspension (200 $\mu 1,2.5 \times 10^{4}$ cells/well) was added to the tissue culture insert in triplicate. They were then transferred into the 24 -well plates containing $700 \mu 1$ conditioned media: i) mock transfection conditioned medium; ii) negative shRNA transfection conditioned medium; iii) fractalkine shRNA transfection conditioned medium. After $8 \mathrm{~h}$ at $37^{\circ} \mathrm{C}$, the inserts were removed away and washed with PBS. Cells that had not migrated (on the upper surface of the inserts) were removed away with cotton swabs. Cells (on the lower surface) were fixed with neutral buffered formalin solution, stained with hematoxylin and eosin (H\&E), and mounted on microscope slides. HUVEC migration was quantitated by counting the number of cells in three random fields (x200) per insert $(3,19,20)$.

In vitro tube formation assay. The effects of conditioned media on HUVEC differentiation were examined by in vitro tube formation on matrigel (BD Biosciences, CA). HUVEC was harvested and diluted (4x10 cells) in $200 \mu 1$ serum-free medium, then seeded on matrigel-coated 24-well plates in triplicate, and incubated at $37^{\circ} \mathrm{C}$ for $1 \mathrm{~h}$. The culture media were then replaced by $700-\mu 1$ conditioned media: i) mock transfection conditioned medium; ii) negative shRNA transfection conditioned medium; iii) fractalkine shRNA transfection conditioned medium. After incubation at $18 \mathrm{~h}$, tube formation images were captured at a magnification of x100 with a digital microscope camera system (Olympus, Tokyo, Japan). The level of the tube formation was quantified by measuring the length of the tubes in three randomly chosen fields from each well using an Image-Pro Plus software $(3,20)$.

In vivo angiogenesis. The matrigel plug assay was performed as described previously with slight modifications. Six-weekold, female BALB/c nude mice were obtained. All animal studies were reviewed and approved by animal care and use committee of Xi'an Jiao Tong University Medical center and are in accordance with the guidelines of the Department of Health and Human Services. Matrigel was mixed with: i) $5 \times 10^{5}$ HepG2 cells transfected with lipofectamine 2000; ii) $5 \times 10^{5}$ HepG 2 cells transfected with negative shRNA; iii) $5 \times 10^{5}$ HepG2 cells transfected with fractalkine shRNA. Matrigel mixtures were injected into the mid-abdominal region of 6-week-old, female BALB/c nude mice. Matrigel rapidly forms a solid gel at body temperature, thus allowing the incorparated substances to be released slowly for prolonged periods of time. Six mice per group were treated

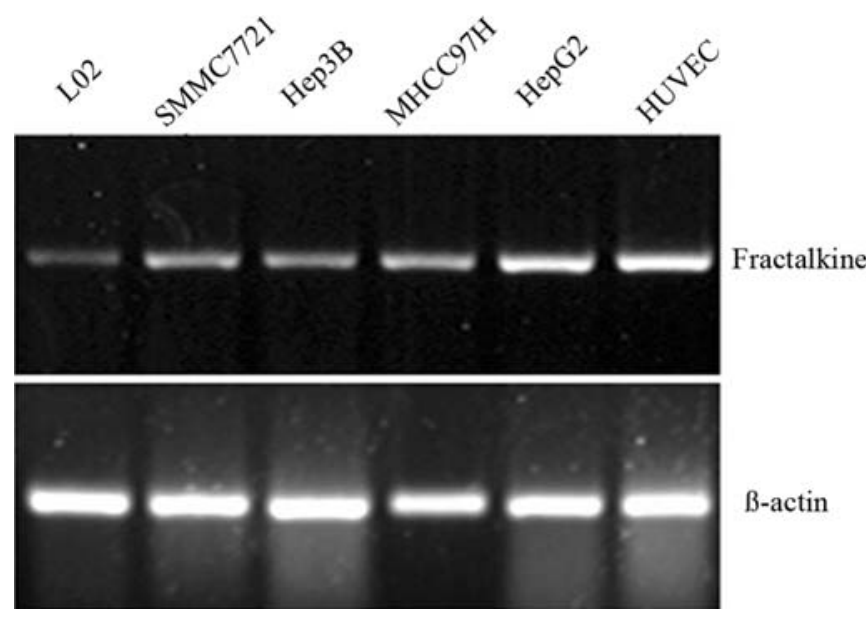

Figure 1. Establishment of hepatocellular carcinoma cell lines expressing fractalkine. Hepatocellular carcinoma cell lines expressed higher fractalkine mRNA comparing with normal liver cell line L02. HUVEC was used as a positive control. Meanwhile, the HepG2 cells expressed higher fractalkine than other hepatocellular carcinoma cells. $\beta$-actin used as a internal control.

for 10 days. The animals were then euthanized and the matrigel plugs removed. The matrigel plugs were fixed in neutral buffered formalin solution and embedded in paraffin. Five micrometer thick sections were stained with hematoxylin and eosin (H\&E). Quantitative analysis of angiogenesis in the matrigel plugs was performed using Image-Pro Plus software. For each plug, three randomly chosen fields were assessed and the average area occupied by microvessels was calculated $(3,20,21)$.

Statistical analysis. All statistical analysis was performed using SPSS13.0 software. The results are presented as mean $\pm \mathrm{SD}$ of three replicated assays. Differences between the groups were analyzed using the Student's t-test. A P-value $<0.05$ was considered to be statistically significant.

\section{Results}

Establishment of hepatocellular carcinoma cell lines expressing fractalkine. In order to establish the role of fractalkine in hepatocellular carcinoma, we used RT-PCR to detect the expression of fractalkine in hepatocellular carcinoma cell lines: HepG2, Hep3B, MHCC97H, SMMC7721. As shown in Fig. 1, hepatocellular carcinoma cell lines expressed higher levels of fractalkine mRNA compared to the normal liver cell line L02. HepG2 cells expressed higher levels of fractalkine than other hepatocellular carcinoma cell lines. HUVEC was used as a positive control. The housekeeping gene $\beta$-actin was used as a loading control.

Knockdown of fractalkine gene expression in HepG2 cells by shRNA-mediated RNAi. Knockdown of transcripts using shRNA is a powerful tool for studying gene function. To inhibit fractalkine gene expression, we developed pGPU6/ GFP/Neo vectors containing small hairpin constructs capable of generating 19 nucleotide duplex RNAi oligonucleotides. 
A
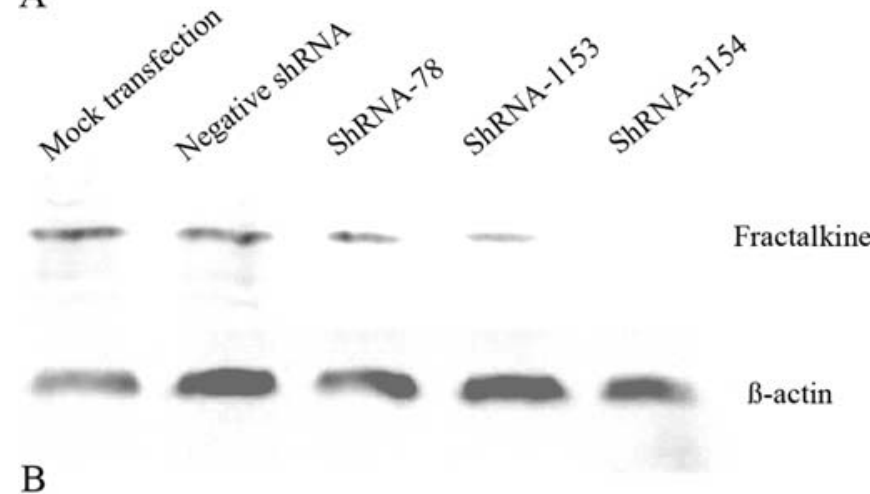

B

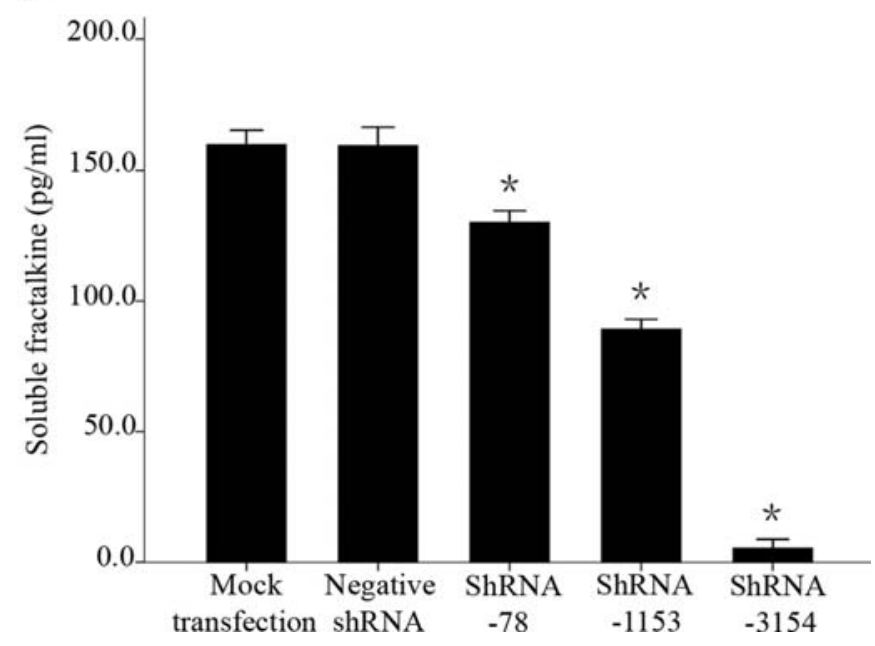

Figure 2. Effect of shRNA mediated RNAi targeting of fractalkine at the protein level. (A) Fractalkine suppression of the membrane-anchored form, as detected by Western blot analysis. (B) ELISA analysis showed the inhibition of the soluble fractalkine in the conditioned media. $* \mathrm{P}<0.05$.

We succeeded in obtaining stable shRNA vector-transfected cells termed: shR-78 (target position, 78), shR-1153 (target position, 1153), shR-3154 (target position, 3154) and negative shRNA (negative control). Western blot analysis showed that compared with negative shRNA transfection and mock transfection, the expression of membrane-anchored fractalkine was inhibited in fractalkine shRNA transfection, especially in shRNA-3154 $(\mathrm{P}<0.05)$ (Fig. 2A); soluble fractalkine was reduced from $159.8 \pm 2.8$ in mock transfection and 158.5 \pm 3.5 in negative control to $5.3 \pm 1.7$ in shRNA-3154 transfection $(\mathrm{P}<0.05)$ (Fig. 2B).

Effect of conditioned media on HUVEC proliferation and migration. HUVEC proliferation and migration are crucial steps in angiogenesis. The first step in angiogenesis may be the secretion of angiogenic factors, promoting endothelial cell proliferation and migration, and the formation of new vessels which sprout from pre-existing vessels $(22,23)$. To test our hypothesis that inhibition of fractalkine leads to the suppression of angiogenesis in HepG2 cells, we first investigated the effect of conditioned media on the proliferation and migration of HUVEC. We collected the condition media from HepG2 cells to examine the effect of suppression of fractalkine on tumor-induced angiogenesis. HepG2 cells were transfected with lipofectamine 2000,
A
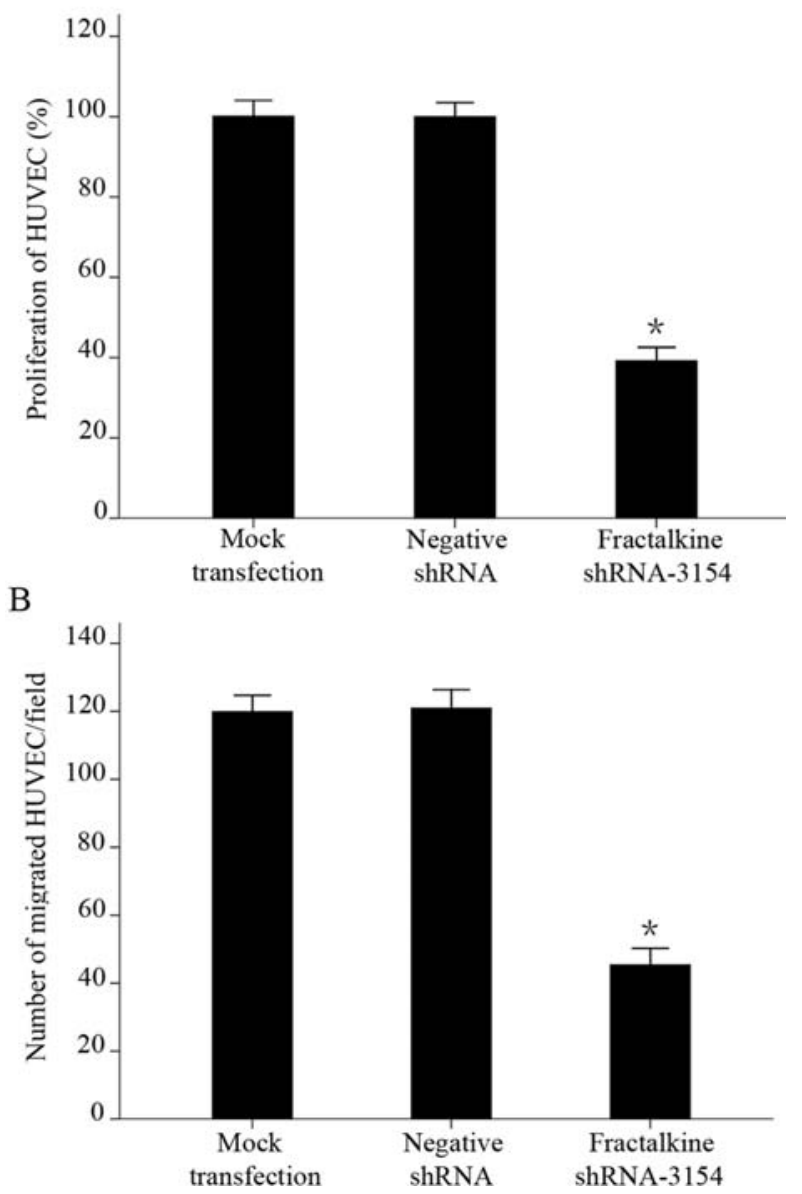

Figure 3. Effects of conditioned media on HUVEC proliferation and migration. (A) Effects on proliferation. HUVEC was seeded in 96-well plates in triplicate and incubated with mock transfection conditioned medium, negative shRNA transfection conditioned medium and fractalkine shRNA3154 transfection conditioned medium. After $24 \mathrm{~h}$, HUVEC proliferation was detected by MTT assay. The values indicate the mean \pm SD of triplicate experiments. ${ }^{*} \mathrm{P}<0.05$. (B) Effects on migration. Migration assay was performed in an invasion chamber. The lower chambers were added with mock transfection conditioned medium, negative shRNA conditioned medium and fractalkine shRNA-3154 conditioned medium. The upper chamber was seeded by HUVEC in triplicate and cultured for $8 \mathrm{~h}$. Non-migrated cells on the upper surface of inserts were removed and migrated cells were quantified under a microscope $(\mathrm{x} 200) .{ }^{*} \mathrm{P}<0.05$.

negative shRNA and fractalkine shRNA-3154 then incubated for $48 \mathrm{~h}$, and culture supernatants were collected. They were labeled as mock transfection medium, negative shRNA medium and fractalkine shRNA-3154 medium.

HUVEC proliferation was inhibited by the conditioned medium obtained from fractalkine shRNA-3154 treated HepG2 cells compared with the mock transfection and the negative shRNA transfection treated cells $(\mathrm{P}<0.05)$ (Fig. 3A). Also, the number of migrated HUVECs was significantly reduced in conditioned medium obtained from fractalkine shRNA-3154 treated HepG2 cells $(\mathrm{P}<0.05)$ compared with the mock transfection and the negative shRNA (Fig. 3B).

Effect of down-regulation of HCC-associated angiogenesis by fractalkine suppression in vitro and in vivo. As shown in Fig. 4, mock transfection and negative shRNA tansfection conditioned medium induced tube formation and cordlike 
A

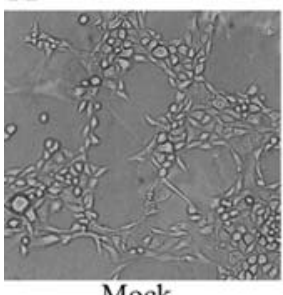

Mock

transfection

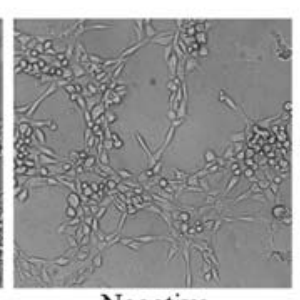

Negative

shRNA

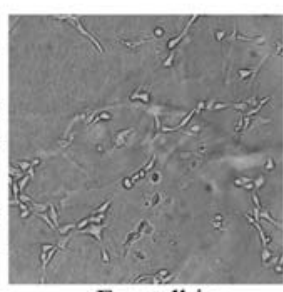

Fractalkine shRNA-3154

B

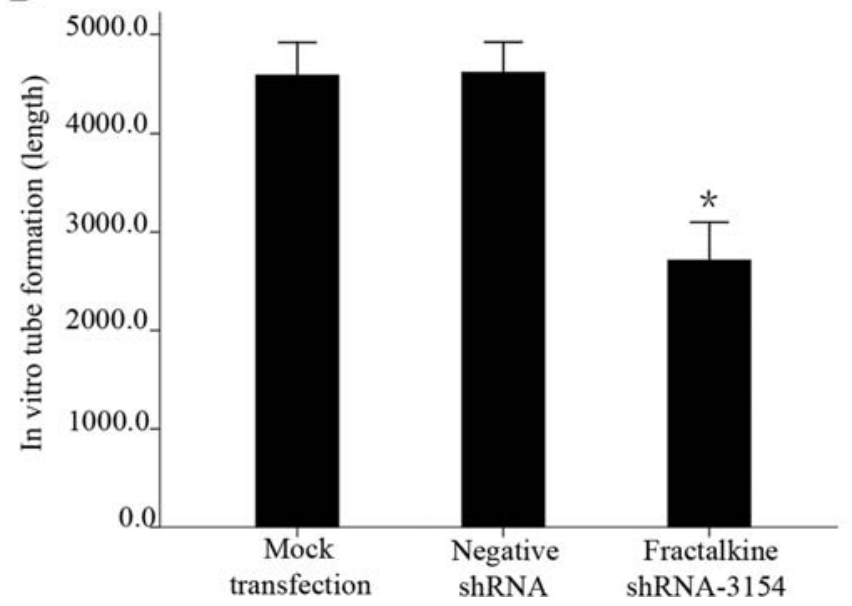

Figure 4. Effect of conditioned media on HUVEC tube formation. (A) Representative phase-contrast micrograph of tube formation (x100). HUVEC was seeded in triplicate on the matrigel-coated 24-well plates and incubated for $18 \mathrm{~h}$ with mock transfection conditioned medium, negative control conditioned medium and fractalkine shRNA-3154 transfection conditioned medium. (B) The mean tube length is shown. ${ }^{*} \mathrm{P}<0.05$.

structures. On the other hand, cultured with fractalkine shRNA-3154 transfection conditioned medium resulted in short and blunt tubes and cordlike structures. Quantitative measurements confirmed that the fractalkine shRNA-3154 caused a significant decrease up to $41 \%$ in mean tube length in vitro compared with mock transfection and negative shRNA $(\mathrm{P}<0.05)$. In Fig. 5, histological and quantitative measurements further demonstrated that fractalkine shRNA-3154 significantly reduced the angiogenic response in vivo. The mean area of microvessels decreased from $1181.6 \pm 33.2 \mu \mathrm{m}^{2}$ in the mock transfection and $117929.9 \mu \mathrm{m}^{2}$ in the negative shRNA to $727.5 \pm 29.2 \mu \mathrm{m}^{2}$ in the fractalkine shR-3154 transfection group $(\mathrm{P}<0.05)$.

\section{Discussion}

The chemokines, initially identified in inflamed tissues, are a superfamily of small secreted peptides that are characterized by their ability to regulate the recruitment and trafficking of leukocytes via chemoattraction (24). Additional roles for chemokines are also recognized, including the regulation of haematopoietic precursors, angiogenesis, and regulation of extracellular matrix (25). In normal physiology, chemokines have pleiotrophic effects; therefore, it stands to reason that in cancer chemokines will also have wide-ranging functions. In fact, compelling evidence has revealed the multifunctional role of the chemokine network in cancer; they may act as

A
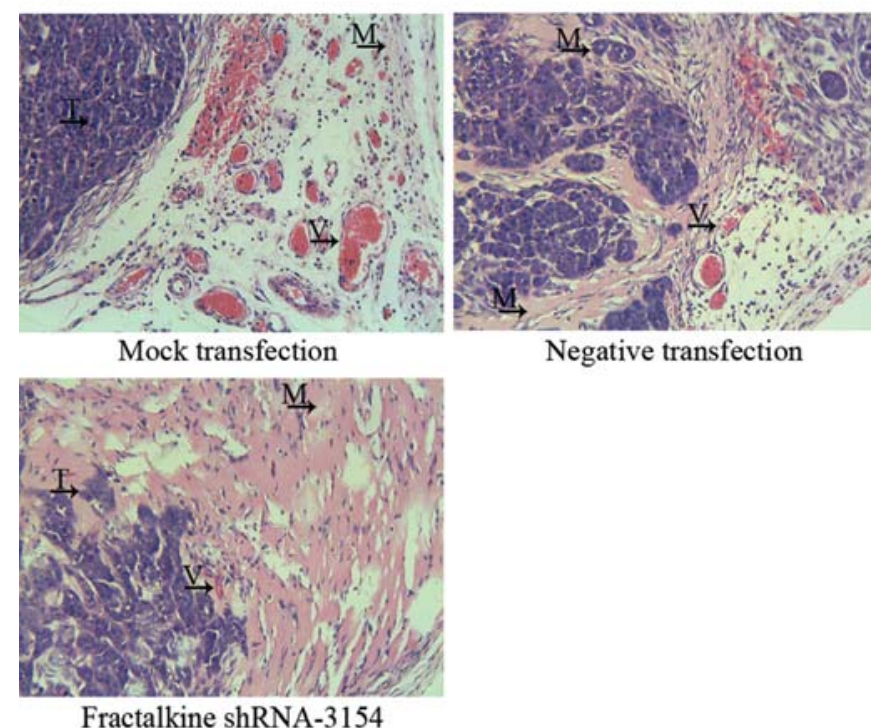

Fractalkine shRNA-3154

B

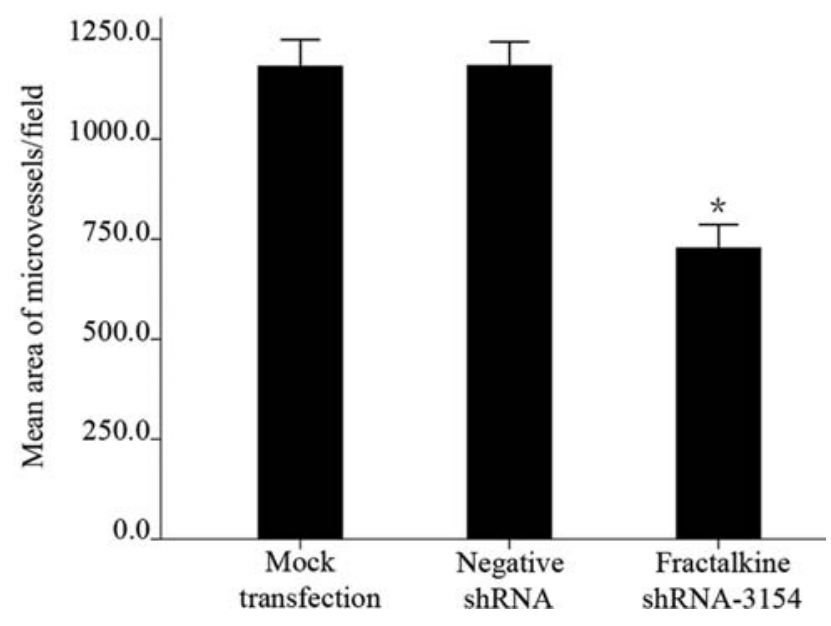

Figure 5. In vivo angiogenesis assay. (A) Microscopic examination of the matrigel plugs, hematoxylin and eosin stained sections of the plugs (x200). T, tumor cells; V, vessels; M, matrigel. (B) The mean area occupied by microvessels per field is shown. ${ }^{*} \mathrm{P}<0.05$.

growth or survival factors, regulate angiogenesis, determine metastatic spread, and controlling leukocyte infiltration into tumors to hinder the antitumor immune responses $(26,27)$.

Many studies have suggested that chemokines and their receptors are involved in the the progression of hepatocellular carcinoma $(28,29)$, which develops following hepatitis and liver fibrosis. Chronic inflammation, together with irritants, enhances cell proliferation. Sustained cell proliferation in an environment rich in inflammatory cells, growth factors, DNA damage promoting agents, and activated stroma promoting the risk of neoplasm formation (30). The common features shared by cancer and inflammation, such as angiogenesis, have been highlighted recently by attempts to treat cancer by targeting the inflammatory response $(31,32)$. Inflammatory cells, cytokines, and chemokines in liver cancers affect the stromal microenvironment, suggesting that inflammation and cancer may be interrelated through the angiogenic process. In this respect, 
special attention should be placed on the essential role of chemokines in carcinogenesis and angiogenesis.

Fractalkine exists as a membrane bound molecular and soluble form released via cleavage at a proximal membrane site. Fractalkine and its receptor are expressed on endothelial cells and activated by inflammatory cytokines, such as interferon- $\gamma$, tumor necrosis factor- $\alpha$, and interleukin-1 (33).

Efsen et al observed that both fractalkine and CX3CR1 are up-regulated during chronic injury, in areas of portal and lobular inflammation (14). In severe acute hepatitis, fractalkine and CX3CR1 are expressed at high levels both at in areas of inflammation and in regenerating epithelial cells. We suggest that hepatocellular carcinoma cells acquire a stable expression of fractalkine during chronic inflammation. At the early stages of carcinoma, high-expression cancer cells promote the adhesion to each other, and, through cross-talk, induce angiogenesis and proliferation. At the later stages of carcinoma, in order mediate metastasis, liver cancer cells highly express the tumor necrosis factor- $\alpha$-converting enzyme (also known as ADAM17) and ADAM10 that mediates the cleavage and shedding of fractalkine, and reduce the adhesive power between cancer cells $(34,35)$.

Our studies suggest liver cancer cell lines stably express fractalkine. We have used gene silencing technology to knock down the expression of fractalkine, and consequently reduce the angiogenesis in hepatocellular carcinomas. This further establishes that fractalkine is involved in angiogenesis, which is involved in the development of cancer. This result is concordance with former studies. In patients and rats with rheumatoid arthritis, synovial tissue, macrophages, fibroblasts, endothelial cells, and dendritic cells express fractalkine and CX3CR1 $(36,37)$. Furthermore, fractalkine in synovial fluid from patients with rheumatoid arthritis promotes angiogenic activity in vitro, and the level of fractalkine in the vitreous humor was elevated in patients with proliferative diabetic retinopathy. Fractalkine induce migration of endothelial cells and the formation of endothelial cell capillary tubes on a synthetic matrix. Fractalkine also induced more blood vessel growth than the controls in the rabbit corneal pocket neovascularization assay. Ren also suggested that down regulation of fractalkine can reduce tumor growth in mice (38).

However, the functional role of fractalkine in the progression of cancer is still controversial. Ohta showed that a higher level of expression of fractalkine in patients with colorectal cancer correlates with a higher density of tumorinfiltrating immune cells and results in a better prognosis than in those with a weak expression of fractalkine (39). Vitale et al developed a C26 colon cancer cell line expressing the native fractalkine. In murine models of skin tumors as well as liver and pulmonary metastasis, native fractalkine expression by $\mathrm{C} 26$ colon cancer cells drastically reduced their overall metastatic potential and growth in the target organs (40). Vaccination of mice with lung carcinoma cells gene-modified with fractalkine induced a potent anti-tumor response, which involved chemoattraction of natural killer cells into tumor sites (41). This suggests that fractalkine is an anti-tumor factor; this is contrary to our results suggesting that it is an angiogenic factor. We suggest that fractalkine may be a double-edged sword: the secreted form reproduces many of the effects of wild-type fractalkine, while the membrane-bound form exerts opposing effects, varing from tumor suppression to enhancement, depending on the target tissue and the experimental model. The overall effect of fractalkine results from a critical balance between the activity of the secreted and membrane-anchored forms.

Altogether, our findings provide further insights into the mechanism of angiogenesis in human hepatocellular carcinoma and unequivocally underscore the importance of using animal models that closely mimic the physiopathological parameters of the cancer under study to investigate novel anticancer strategies.

\section{References}

1. Parkin DM, Bray F, Ferlay J and Pisani P: Estimating the world cancer burden: Globocan 2000. Int J Cancer 94: 153-156, 2001.

2. Bruix $J$ and Llovet JM: Prognostic prediction and treatment strategy in hepatocellular carcinoma. Hepatology 35: 519-524, 2002.

3. Mu H, Ohashi R, Yan SY, et al: Adipokine resistin promotes in vitro angiogenesis of human endothelial cells. Cardiovasc Res 70: 146-157, 2006.

4. Poon RT, Ng IO, Lau C, et al: Serum vascular endothelial growth factor predicts venous invasion in hepatocellular carcinoma: a prospective study. Ann Surg 233: 227-235, 2001.

5. Lee TK, Poon RT, Yuen AP, Man K, Yang ZF, Guan XY and Fan ST: Rac activation is associated with hepatocellular carcinoma metastasis by up-regulation of vascular endothelial growth factor expression. Clin Cancer Res 12: 5082-5089, 2006.

6. Kimura YN, Watari K, Fotowati A, et al: Inflammatory stimuli from macrophages and cancer cells synergistically promote tumor growth and angiogenesis. Cancer Sci 98: 2009-2018, 2007.

7. Zlotnik A and Yoshie O: Chemokines: a new classification system and their role in immunity. Immunity 12: 121-127, 2000.

8. Locati MK, Otero K, Schioppa T, et al: The chemokine system: tuning and shaping by regulation of receptor expression and coupling in polarized responses. Allergy 57: 972-982, 2002.

9. Raman D, Baugher PJ, Thu YM and Richmond A: Role of chemokines in tumor growth. Cancer Lett 256: 137-165, 2007.

10. Bazan JF, Bacon KB, Hardiman G, et al: A new class of membrane-bound chemokine with a $\mathrm{CX}(3) \mathrm{C}$ motif. Nature 385: 640-644, 1997.

11. Pan Y, Lloyd C, Zhou H, et al: Neurotactin, a membraneanchored chemokine upregulated in brain inflammation. Nature 387: 611-617, 1997.

12. Papadopoulos EJ, Sassetti C, Saeki H, et al: Fractalkine, a $\mathrm{CX} 3 \mathrm{C}$ chemokine, is expressed by dendritic cells and is upregulated upon dendritic cell maturation. Eur J Immunol 29: 2551-2559, 1999.

13. Lucas AD, Chadwick N, Warren BF, Jewell DP, Gordon S, Powrie F and Greaves DR: The transmembrane form of the CX3CL1 chemokine fractalkine is expressed predominantly by epithelial cells in vivo. Am J Pathol 158: 855-866, 2001.

14. Efsen E, Grappone C, Defranco RM, et al: Up-regulated expression of fractalkine and its receptor CX3CR1 during liver injury in humans. J Hepatol 37: 39-47, 2002.

15. Umehara H, Goda S, Imai T, et al: Fractalkine, a CX3Cchemokine, functions predominantly as an adhesion molecule in monocytic cell line THP-1. Immunol Cell Biol 79: 298-302, 2001.

16. Volin MV, Woods JM, Amin MA, Harlow LA and Koch AE: Fractalkine: a novel angiogenic chemokine in rheumatoid arthritis. Am J Pathol 159: 1521-1530, 2001.

17. You JJ, Yang CH, Huang JS, Chen MS and Yang CM: Fractalkine, a CX3C chemokine, as a mediator of ocular angiogenesis. Invest Ophthalmol Vis Sci 48: 5290-5298, 2007.

18. Wu XF, Fan J, Wang XY, et al: Downregulation of CCR 1 inhibits human hepatocellular carcinoma cell invasion. Biochem Biophys Res Commun 355: 866-871, 2007.

19. Albini A, Florio T, Giunciuglio D, et al: Somatostatin controls Kaposi's sarcoma tumor growth through inhibition of angiogenesis. FASEB J 13: 647-655, 1999.

20. Fu YG, Sung JJY, Wu KC, et al: Inhibition of gastric cancerassociated angiogenesis by antisense COX-2 transfectants. Cancer Lett 224: 243-252, 2005. 
21. Lee, YS, Yang HO, Shin KH, et al: Suppression of tumor growth by a new glycosaminoglycan isolated from the African giant snail Achatina fulica. Eur J Pharmacol 465: 191-198, 2003.

22. Folkman J, Bach M, Rowe JW, et al: Tumor angiogenesis, therapeutic implications. New Engl J Med 285: 1182, 1971.

23. Folkman J: Angiogenesis in cancer, vascular, rheumatoid and other disease. Nat Med 1: 27-31, 1995.

24. Zlotnik A: Chemokines and cancer. Int J Cancer 119: 2026-2029, 2006.

25. Kulbe H, Levinson NR, Balkwill F and Wilson JL: The chemokine network in cancer, much more than directing cell movement. Int J Dev Biol 48: 489-496, 2004.

26. Balkwill F: Chemokine biology in cancer. Semin Immunol 15: 49-55, 2003.

27. Slettenaar VIF and Wilson JL: The chemokine network: a target in cancer biology? Adv Drug Deliver Rev 58: 962-974, 2006.

28. Liu YQ, Poon RT, Hughes J, Feng XQ, Yu WC and Fan ST: Chemokine receptors support infiltration of lymphocyte subpopulations in human hepatocellular carcinoma. Clin Immunol 114: 174-182, 2005 .

29. Hirano S, Iwashita Y, Sasaki A, Kai S, Ohta M and Kitano S: Increased mRNA expression of chemokines in hepatocellular carcinoma with tumor-infiltrating lymphocytes. J Gastroen Hepatol 22: 690-696, 2007.

30. Coussens LM and Werb Z: Inflammation and cancer. Nature 420: 860-867, 2002.

31. Balkwill $\mathrm{F}$ and Mantovani A: Inflammation and cancer: back to Virchow? Lancet 357: 539-545, 2001.

32. Pollard JW: Tumour-educated macrophages promote tumour progression and metastasis. Nat Rev Cancer 4: 71-78, 2004.

33. Ahn SY, Cho CH, Park KY, et al: Tumor necrosis factor-alpha induces fractalkine expression preferentially in arterial endothelial cells and mithramycin A suppresses TNF-alpha-induced fractalkine expression. Am J Pathol 164: 1663-1672, 2004.
34. Garton KJ, Gough PJ, Blobel CP, Murphy G, Greaves DR, Dempsey PJ and Raines EW: Tumor necrosis factor-alpha-converting enzyme (ADAM17) mediates the cleavage and shedding of fractalkine (CX3CL1). J Biol Chem 276: 37993-38001, 2001.

35. Hundhausen C, Misztela D, Berkhout TA, et al: The disintegrinlike metalloproteinase ADAM10 is involved in constitutive cleavage of CX3CL1 (fractalkine) and regulates CX3CL1mediated cell-cell adhesion. Blood 102: 1186-1195, 2003.

36. Ruth JH, Rottman JB, Katschke KJ, et al: Selective lymphocyte chemokine receptor expression in the rheumatoid joint. Arthritis Rheum 44: 2750-2760, 2001

37. Ruth JH, Volin MV, Haines GK, et al: Fractalkine, a novel chemokine in rheumatoid arthritis and in rat adjuvant-induced arthritis. Arthritis Rheum 44: 1568-1581, 2001.

38. Ren T, Chen Q, Tian ZG and Wei HM: Down-regulation of surface fractalkine by RNA interference in B16 melanoma reduced tumor growth in mice. Biochem Biophys Res Commun 364: 978-984, 2007.

39. Ohta M, Tanaka F, Yamaguchi H, Sadanaga N, Inoue H and Mori M: The high expression of Fractalkine results in a better prognosis for colorectal cancer patients. Int J Oncol 26: 41-47, 2005.

40. Vitale S, Cambien B, Karimdjee BF, et al: Tissue-specific differential antitumour effect of molecular forms of fractalkine in a mouse model of metastatic colon cancer. Gut 56: 365-372, 2007.

41. Guo J, Chen TY, Wang BC, et al: Chemoattraction, adhesion and activation of natural killer cells are involved in the anti-tumor immune response induced by fractalkine/CX3CL1. Immunol Lett 89: 1-7, 2003. 